Original article

\title{
The predictors of high titer of anti-SARS-CoV-2 antibody of convalescent plasma donors
}

\author{
Shinta Oktya Wardhani ${ }^{\text {a, }{ }^{* *}, \text { Jonny Karunia Fajar }}{ }^{\text {b, }}$, Nina Nurarifah ${ }^{a}$, Djoko Heri Hermanto ${ }^{\text {a }}$ \\ Siti Fatonah ${ }^{\mathrm{c}}$, Susanthy Djajalaksana ${ }^{\mathrm{d}}$, Arie Zainul Fatoni ${ }^{\mathrm{e}}$, Putu Moda Arsana ${ }^{\mathrm{f}}$,

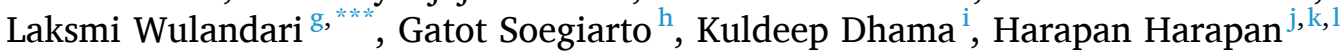 \\ ${ }^{a}$ Division of Hematology and Oncology, Department of Internal Medicine, Faculty of Medicine, Universitas Brawijaya, Malang, 65145, Indonesia \\ ${ }^{\mathrm{b}}$ Brawijaya Internal Medicine Research Center, Department of Internal Medicine, Faculty of Medicine, Universitas Brawijaya, Malang, 65145, Indonesia \\ ${ }^{\mathrm{c}}$ Department Clinical Pathology, Faculty of Medicine, Universitas Brawijaya, Malang, 65145, Indonesia \\ d Department of Pulmonology and Respiratory Medicine, Faculty of Medicine, Universitas Brawijaya, Malang, 65145, Indonesia \\ ${ }^{\mathrm{e}}$ Department of Anesthesiology and Intensive Therapy, Faculty of Medicine, Universitas Brawijaya, Malang 65145, Indonesia \\ ${ }^{\mathrm{f}}$ Division of Endocrinology, Diabetes \& Metabolism, Department of Internal Medicine, Faculty of Medicine, Universitas Brawijaya, Malang, 65145, Indonesia \\ ${ }^{g}$ Department of Pulmonology and Respiratory Medicine, Faculty of Medicine, Universitas Airlangga, Surabaya, 60286, Indonesia \\ ${ }^{\mathrm{h}}$ Division of Allergy \& Immunology, Department of Internal Medicine, Faculty of Medicine, Universitas Airlangga, Surabaya, 60286, Indonesia \\ ${ }^{i}$ Division of Pathology, ICAR-Indian Veterinary Research Institute, Izatnagar, Bareilly, 243 122, Uttar Pradesh, India \\ ${ }^{\mathrm{j}}$ Medical Research Unit, School of Medicine, Universitas Syiah Kuala, Banda Aceh, 23111, Indonesia \\ ${ }^{\mathrm{k}}$ Tropical Disease Centre, School of Medicine, Universitas Syiah Kuala, Banda Aceh, 23111, Indonesia \\ ${ }^{1}$ Department of Microbiology, School of Medicine, Universitas Syiah Kuala, Banda Aceh, 23111, Indonesia
}

\section{A R T I C L E I N F O}

\section{Keywords:}

Convalescent plasma

COVID-19

Donors

Predictor

Titers of antibody

\begin{abstract}
A B S T R A C T
Background: Recent evidence suggested that the higher titers of anti-severe acute respiratory syndrome coronavirus 2 (SARS-CoV-2) antibody from convalescent plasma donors contributed to the clinical improvement in coronavirus disease 2019 (COVID-19) patients. However, the titers of anti-SARS-CoV-2 antibodies varied in each individual, and the precise factors that might govern such variation have not been elucidated.

Objectives: To assess the factors associated with high titers of anti-SARS-CoV-2 antibody among COVID-19 convalescent plasma (CCP) donors.

Methods: A cross-sectional study was conducted in Saiful Anwar General Hospital, Malang, Indonesia. Information of interest including demographic characteristics, clinical symptoms, comorbidities, laboratory findings, and the titers of anti-SARS-CoV-2 antibody among COVID-19 CCP donors were collected. The correlation was assessed using multiple logistic regression.

Results: A total of 50 COVID-19 CCP donors with the titers of anti-SARS-CoV-2 antibody of more than 1:320 and 33 donors with the titers of less than 1:320 were analyzed. Our analysis revealed that CCP donors with history of cough, fever, dyspnea, and pneumonia significantly had higher titers of anti-SARS-CoV-2 antibody compared to asymptomatic donors. Moreover, CCP donors with elevated levels of eosinophils and immature granulocytes and low levels of albumins had higher levels of anti-SARS-CoV-2 antibody. The titer of antibody was not affected by comorbidities of donors.

Conclusions: CPP donors who had experience of symptomatic COVID-19 with high eosinophils level, high immature granulocytes and low albumin level have higher titers of anti-SARS-COV-2 antibody than those who experienced asymptomatic COVID-19. Our current findings may be used as the additional baseline criteria for selecting the donors of CCP for the management of COVID-19.
\end{abstract}

\footnotetext{
* Corresponding author.

$* *$ Corresponding author.

$* * *$ Corresponding author.

E-mail addresses: shinta_oktya.fk@ub.ac.id (S.O. Wardhani), gembyok@gmail.com (J.K. Fajar), laksmi.wulandari@fk.unair.ac.id (L. Wulandari).
} 


\section{Introduction}

The management of coronavirus disease 2019 (COVID-19) remains a major challenge. Since the consolidation project from World Health Organization (WHO) suggests a weak efficacy of hydroxychloroquine, remdesivir, interferon regimens, and lopinavir to treat COVID- $19^{1}$ and the vaccines are still being developed, a treatment using passive immunization concept might be promising. Convalescent plasma (CCP), a treatment by transferring neutralizing anti-viral antibodies and other immunomodulators via plasma transfusion, had been historically proven to provide the clinical improvement of similar infectious diseases $^{2,3}$; and therefore, CCP is proposed as one of the promising treatments of COVID-19. ${ }^{4}$ However, the use of CCP to treat COVID-19 patients have raised the dilemma due to the different responses among the patients. ${ }^{5}$ A specific setting of CCP administration needs to be validated.

While the US Food and Drug Administration (FDA) has suggested that the titers of anti-SARS-COV-2 antibody of more than $1: 160$ is considered as the effective CCP, ${ }^{6}$ the evidence regarding the outcomes of CCP remained controversial. ${ }^{7}$ Recent studies indicated that the titers of anti-SARS-COV-2 antibody of more than 1:320 from CCP donors provided clinical improvement for treating COVID-19 patients. ${ }^{8,9}$ However, the titers of anti-SARS-CoV-2 antibody varied in each individual, and these titers might be affected by a wide variety of conditions such as host, perinatal, extrinsic, behavioral, and nutritional factors ${ }^{10}$ as well as magnitude of transmission of the COVID-19 in region. ${ }^{11}$ Some studies have been conducted in US and China; ${ }^{12,13}$ however, no study has been conducted in Indonesia to assess the factors of the host and disease course that might affect the titers of anti-SARS-COV-2 antibody. Therefore, this study was conducted to assess the predictors of the titers of anti-SARS-COV-2 antibody among Indonesian CCP donors.

\section{Methods}

\subsection{Study design and patients}

Between July and November 2020, a cross-sectional study was conducted in Saiful Anwar General Hospital, Malang, Indonesia to assess the factors that might affect the titer of anti-SARS-CoV-2 antibody among the donors of CCP. We used total sampling method to recruit the participants. All participants met the criteria for CCP transfusion as recommended by the FDA. ${ }^{6}$ Three investigators contacted each participant privately. Samples were collected from healthy COVID-19 patients who had been asymptomatic for $>14$ days (the 15th day). Participants with any of the following criteria were excluded: pregnant woman, having the history of transfusion reaction, and treated with immunoglobulin in the last 30 days. Our study protocols conformed with the principle outlined in the Declaration of Helsinki and had been approved by Indonesian Health Research Ethics Committee of the National Research and Development Agency (No. LB.02.01/2/KE.351/2020) and all participants provided written informed consent.

\subsection{Outcome measures}

The predictor variables in our study were symptoms, comorbidities, and laboratory findings. Disease symptoms included diarrhea, fever, cough, dysgeusia, dyspnea, and pneumonia. The comorbidities were obesity, overweight, diabetes mellitus, and hypertension. The laboratory findings were hemoglobin, leukocytes, thrombocytes, eosinophils, basophils, neutrophils, lymphocytes, neutrophil-lymphocyte ratio (NLR), immature granulocytes, total proteins, albumins, globulins, and total cholesterols. The outcome was the titer of anti-SARS-CoV-2 antibody, divided into less than and more than 1:320, as recommended by previous studies. ${ }^{8,9}$ CCP was collected by plasmapheresis using Haemonetics MSC $®$ plus (Haemonetics, Boston, Massachusetts, US).

\subsection{Measurement of anti-SARS-CoV-2 antibody}

Blood samples were collected and stored at $-20{ }^{\circ} \mathrm{C}$ and were tested for anti-SARS-CoV-2 IgM and IgG levels using qualitative lateral flow immunochromatographic assay (ICA) (SD Biosensor, Ye Suwon-shi, Korea). The testing was conducted in accordance to the manufacturer's recommendations and the protocols of the previous studies. ${ }^{14,15}$ To determining the antibody titer, two-fold dilutions of plasma (starting at a 1:20 dilution) was carried out. Infectious virus was added to the plasma dilutions at a final concentration of $1 \times 10^{4} \mathrm{TCID}_{50} / \mathrm{mL}(100$ TCID $_{50}$ per $100 \mu \mathrm{L}$ ). The samples were incubated for $1 \mathrm{~h}$ at room temperature, and then $100 \mu \mathrm{L}$ of each dilution was added to 1 well of a 96-well plate of Vero E6-TMPRSS2 cells in sextuplet for $6 \mathrm{~h}$ at $37^{\circ} \mathrm{C}$. The inocula were removed, the fresh media was added, and the plates were incubated at $37{ }^{\circ} \mathrm{C}$ for 2 days. The cells were fixed with $4 \%$ formaldehyde and then stained with Napthol Blue Black (Millipore Sigma, Darmstadt, Germany). The antibody titer was calculated as the highest serum dilution that prevent the cytopathic effect in $50 \%$ of the wells.

\subsection{Statistical analysis}

The association between the predictor covariates and the titers of anti-SARS-COV-2 antibody was performed using multiple logistic regression. All significant tests were two tailed and the $\mathrm{P}$ value $<0.05$ was considered statistically significant. All analyses in our present study were conducted using a Statistical Package of Social Science 17.0 software (SPSS Inc, Chicago, IL).

\section{Results}

A total of 50 participants with the titer of anti-SARS-CoV-2 antibody more than 1:320 and 33 participants with the titers of anti-SARS-CoV-2 antibody less than 1:320 were included in our study. Initially, we included 99 participants; 16 participants were excluded due to pregnant status, having the history of transfusion reaction, and having the history of immunoglobulin therapy in the last 30 days. The baseline characteristics of participants are presented in Table 1.

Our findings suggested that the history of fever, cough, dyspnea, and pneumonia among the CCP donors was associated with increased titer of anti-SARS-CoV-2 antibody compared to asymptomatic patients. Our study also found that participants with the high level of eosinophiles and immature granulocytes, and low albumins level were associated with high titers of anti-SARS-CoV-2 antibody.

\section{Discussion}

Our study found that donors that experienced symptomatic COVID19 had higher levels of anti-SARS-CoV-2 antibody compared to those with asymptomatic participants. Our study conformed with the previous studies that found symptomatic CCP donors were associated with high titer of anti-SARS-CoV-2 antibody. ${ }^{12,13}$ The report assessing the potential factors on the titers of antibody was limited, and therefore, the speculation theory in this context remained controversial. A recent clinical evidence showed that high antibody titer was found in critically ill COVID-19 patients than non-critically ill patients. ${ }^{16}$ Moreover, patients with high level of C-reactive protein (CRP), low level of lymphocytes, and old age, which are commonly found in severe COVID-19 patients, were associated with high level of anti-SARS-CoV-2 antibody. ${ }^{17}$

It was proposed that the binding of specific antibody to the virus through $\mathrm{Fc} \gamma$ receptors (Fc $\gamma \mathrm{Rs}$ ) which are highly expressed in lung epithelial cells may facilitate the entry of virus into immune cells. ${ }^{18}$ The evidence also revealed that immunoglobulin therapy by blocking Fc $\gamma$ Rs was considered the potential therapy in COVID-19 patients. ${ }^{19}$ These evidences suggest that disease progression might play a pivotal role to govern the titers of antibody. Therefore, in our current study, those individual donors experienced with more predominant symptomatic 
Table 1

Baseline characteristics of patients included in our analysis.

\begin{tabular}{|c|c|c|c|c|c|}
\hline \multirow[t]{2}{*}{ Characteristics } & \multicolumn{2}{|l|}{ Antibody titers } & \multirow[t]{2}{*}{ OR } & \multirow[t]{2}{*}{$95 \% \mathrm{CI}$} & \multirow[t]{2}{*}{$\mathrm{p}$} \\
\hline & $>1: 320(\mathrm{n}=50)$ & $<1: 320(\mathrm{n}=33)$ & & & \\
\hline Age (years) & $37(23-55)$ & 35 (19-59) & 1.18 & $0.53-2.63$ & 0.679 \\
\hline Male, n (\%) & $38(76.0)$ & $21(63.6)$ & 1.81 & $0.69-4.73$ & 0.227 \\
\hline \multicolumn{6}{|l|}{ Symptoms, n (\%) } \\
\hline Diarrhea & $9(18.0)$ & $5(15.2)$ & 1.23 & $0.37-4.06$ & 0.735 \\
\hline Fever & $46(92.0)$ & $6(18.2)$ & 51.75 & 13.40-199.91 & $<0.0001$ \\
\hline Cough & $45(90.0)$ & $3(9.1)$ & 90.00 & $20.00-404.99$ & $<0.0001$ \\
\hline Dysgeusia & $6(12.0)$ & $3(9.1)$ & 1.36 & $0.32-5.88$ & 0.677 \\
\hline Dyspnea & $35(70.0)$ & $1(3.0)$ & 74.67 & $9.33-597.81$ & $<0.0001$ \\
\hline Pneumonia & $32(65.3)$ & $1(3.0)$ & 56.89 & $7.16-451.93$ & $<0.0001$ \\
\hline \multicolumn{6}{|l|}{ Comorbidity, n (\%) } \\
\hline No comorbidity & $39(78.0)$ & $30(90.9)$ & 0.36 & $0.09-1.39$ & 0.136 \\
\hline Obesity & $4(8.0)$ & $1(3.0)$ & 2.78 & $0.30-26.07$ & 0.370 \\
\hline Overweight & $2(4.0)$ & $0(0.0)$ & 3.45 & $0.16-74.26$ & 0.429 \\
\hline Diabetes & $1(2.0)$ & $2(6.1)$ & 0.32 & $0.03-3.64$ & 0.356 \\
\hline Hypertension & $4(8.0)$ & $0(0.0)$ & 6.48 & $0.34-124.54$ & 0.215 \\
\hline \multicolumn{6}{|l|}{ Laboratory findings } \\
\hline Hemoglobin (gr/dl) & $14.7(11.1-17.1)$ & 14.5 (12.1-16.7) & 1.00 & $0.45-2.22$ & 1.000 \\
\hline Leukocytes $(/ \mu \mathrm{l})$ & $6980(4750-11570)$ & $6650(4320-13440)$ & 1.14 & $0.51-2.53$ & 0.748 \\
\hline Thrombocyte $\left(\mathrm{x} 10^{3} / \mathrm{mm}^{3}\right)$ & $276(66-430)$ & $258(66-429)$ & 1.64 & $0.74-3.65$ & 0.226 \\
\hline Eosinophils (Abs) & $0.19(0.02-0.42)$ & $0.17(0.01-0.61)$ & 5.06 & $2.20-11.66$ & $<0.0001$ \\
\hline Basophils (Abs) & $0.03(0.00-0.09)$ & $0.03(0.01-0.07)$ & 1.00 & $0.45-2.22$ & 1.000 \\
\hline Neutrophils (Abs) & $3.76(1.58-8.60)$ & $3.70(2.17-9.98)$ & 1.06 & $0.48-2.36$ & 0.884 \\
\hline Lymphocytes (Abs) & $2.36(1.44-3.81)$ & $2.23(1.16-3.85)$ & 1.19 & $0.54-2.65$ & 0.665 \\
\hline NLR & $1.62(0.52-4.32)$ & $1.60(0.89-4.67)$ & 1.48 & $0.67-3.30$ & 0.334 \\
\hline Immature granulocytes & $0.03(0.01-0.10)$ & $0.02(0.01-0.07)$ & 6.13 & $2.64-14.26$ & $<0.0001$ \\
\hline Total proteins (gr/dl) & $7.73 \pm 0.46$ & $7.85 \pm 0.41$ & 0.61 & $0.27-1.36$ & 0.227 \\
\hline Albumins (gr/dl) & $4.70 \pm 0.34$ & $4.86 \pm 0.30$ & 0.41 & $0.18-0.92$ & 0.030 \\
\hline Globulins (gr/dl) & $3.03 \pm 0.34$ & $2.99 \pm 0.37$ & 1.23 & $0.55-2.73$ & 0.613 \\
\hline Total cholesterols (mg/dl) & $199(136-343)$ & 203 (127-389) & 0.93 & $0.42-2.07$ & 0.866 \\
\hline
\end{tabular}

Note, data were presented as mean \pm SD or $\mathrm{n}(\%)$; NLR, neutrophil-lymphocyte ratio; OR, odd ratio, CI, confidence interval.

COVID-19 had higher level of anti-SARS-CoV-2 antibody than those asymptomatic donors of CCP.

Our data also suggested that COVID-19 patients with elevated level of eosinophiles and immature granulocytes, and low albumin level were associated with high level of anti-SARS-CoV-2 antibody. There is no study available as comparison of our present findings. The underlying mechanism of our findings might be due to the fact that those laboratory findings are commonly found in severe COVID-19 patients, ${ }^{20,21}$ and high anti-SARS-CoV-2 antibody was associated with severe COVID-19 patients. ${ }^{16}$ Moreover, in the case of viral infection, high level of eosinophils was also suggested to associate with elevated level of antibody. ${ }^{22}$ An increased level of immature granulocytes was also correlated with high risk of viral infection and elevated level of antibody. ${ }^{23}$ Low level of albumin might be affected by the clearance mechanism of antibody, suggesting that negative association might underly between albumin level and titer of anti-SARS-CoV-2 antibody. ${ }^{24}$

To the best of our knowledge, our study is the first report regarding the predictors of anti-SARS-CoV-2 antibody titer in Indonesia. Our current study might provide the baseline data for selecting CCP donors for COVID-19. We expected that a Medical Council should elaborate the precise guideline to determine the prominent candidate of CCP donors for the management of COVID-19. However, further studies are warranted to investigate other predictors to predict high titer of anti-SARSCoV-2 antibody in CCP donors.

Some limitations also need to be discussed. First, we did not involve some potential confounding factors, including genetics, prenatal factors, preexisting immunity, geographic location, family size, smoking, alcohol consumption, exercise, and sleep. ${ }^{10}$ Second, a small sample size in our current study should be interpreted with caution. Third, our current study was cross-sectional which is observational in nature and therefore unable to determine the holistic cause-effect relationship.

\section{Conclusion}

Our study suggests that symptomatic COVID-19 individuals with elevated levels of eosinophiles and immature granulocytes, and low levels of albumins are the prominent predictors of high titer of antiSARS-CoV-2 antibody in CCP donors. Our current study may provide the additional criteria for selecting the CCP donors for the management of COVID-19.

\section{Ethics approval and consent to participate}

Participants had provided written informed consent prior to involve in the study. Our study had been approved by local ethical committee (No. LB.02.01/2/KE.351/2020).

\section{Availability of data and materials}

Data used in our study were presented in the main text.

\section{Consent for publication}

Not applicable.

\section{Funding source}

This study received no external funding.

\section{Authors contributions}

Idea/concept: SOW, JKF. Design: SOW, JKF. Control/supervision: SOW, PMA, LW, GS, KD, HH. Data collection/processing: NN, DHH, SF, SD, AZF. Extraction/Analysis/interpretation: JKF. Literature review: SOW, JKF, LW, GS, KD, HH. Writing the article: JKF. Critical review: LW, GS, KD, HH. All authors have critically reviewed and approved the final 
draft and are responsible for the content and similarity index of the manuscript.

\section{Declaration of competing interest}

None.

\section{Acknowledgement}

\section{We thank to RSUD dr. Saiful Anwar for supporting this project.}

\section{References}

1 Consortium WHOST, Pan H, Peto R, et al. Repurposed antiviral drugs for covid-19 interim WHO solidarity trial results. N Engl J Med. 2020;384(6):497-511.

2 Arabi YM, Hajeer AH, Luke T, et al. Feasibility of using convalescent plasma immunotherapy for MERS-CoV infection, Saudi arabia. Emerg Infect Dis. 2016;22: $1554-1561$.

3 Soo YO, Cheng Y, Wong R, et al. Retrospective comparison of convalescent plasma with continuing high-dose methylprednisolone treatment in SARS patients. Clin Microbiol Infect. 2004;10:676-678.

4 Sun M, Xu Y, He H, et al. A potentially effective treatment for COVID-19: a systematic review and meta-analysis of convalescent plasma therapy in treating severe infectious disease. Int J Infect Dis. 2020;98:334-346.

5 Simonovich VA, Burgos Pratx LD, Scibona P, et al. A randomized trial of convalescent plasma in covid-19 severe pneumonia. N Engl J Med. 2020;384(7):619-629.

6 FDA. Recommendations for Investigational COVID-19 Convalescent Plasma. US Food \& Drugs Administration: US Food \& Drugs Administration; 2020.

7 Abolghasemi H, Eshghi P, Cheraghali AM, et al. Clinical efficacy of convalescent plasma for treatment of COVID-19 infections: results of a multicenter clinical study. Transfus Apher Sci. 2020;59, 102875.

8 Islam A, Rafiq S, Karim S, Laher I, Rashid H. Convalescent plasma therapy in the treatment of COVID-19: practical considerations: Correspondence. Int J Surg. 2020; 79:204-205.

9 Liu STH, Lin HM, Baine I, et al. Convalescent plasma treatment of severe COVID-19: a propensity score-matched control study. Nat Med. 2020;26:1708-1713.
10 Zimmermann $\mathrm{P}$, Curtis N. Factors that influence the immune response to vaccination. Clin Microbiol Rev. 2019;32.

11 Arnold BF, van der Laan MJ, Hubbard AE, et al. Measuring changes in transmission of neglected tropical diseases, malaria, and enteric pathogens from quantitative antibody levels. PLoS Neglected Trop Dis. 2017;11, e0005616.

12 Madariaga MLL, Guthmiller JJ, Schrantz S, et al. Clinical predictors of donor antibody titre and correlation with recipient antibody response in a COVID-19 convalescent plasma clinical trial. J Intern Med. 2020;289(4):559-573.

13 Chen W, Zhang J, Qin X, et al. SARS-CoV-2 neutralizing antibody levels are correlated with severity of COVID-19 pneumonia. Biomed Pharmacother. 2020;130, 110629.

14 Klein SL, Pekosz A, Park HS, et al. Sex, age, and hospitalization drive antibody responses in a COVID-19 convalescent plasma donor population. J Clin Invest. 2020; 130:6141-6150.

15 Gomaa MR, Kandeil A, Mostafa A, et al. Prevalence of severe acute respiratory syndrome coronavirus 2 neutralizing antibodies in Egyptian convalescent plasma donors. Front Microbiol. 2020;11, 596851.

16 Liu W, Fontanet A, Zhang PH, et al. Two-year prospective study of the humoral immune response of patients with severe acute respiratory syndrome. J Infect Dis. 2006;193:792-795.

17 Wu F, Wang A, Liu M, et al. Neutralizing Antibody Responses to SARS-CoV-2 in a COVID-19 Recovered Patient Cohort and Their Implications. 2020.

18 Spiekermann GM, Finn PW, Ward ES, et al. Receptor-mediated immunoglobulin G transport across mucosal barriers in adult life: functional expression of FcRn in the mammalian lung. J Exp Med. 2002;196:303-310.

19 Fu Y, Cheng Y, Wu Y. Understanding SARS-CoV-2-mediated inflammatory responses: from mechanisms to potential therapeutic tools. Virol Sin. 2020;35:266-271.

20 Ponti G, Maccaferri M, Ruini C, Tomasi A, Ozben T. Biomarkers associated with COVID-19 disease progression. Crit Rev Clin Lab Sci. 2020;57:389-399.

21 Mudatsir M, Fajar JK, Wulandari L, et al. Predictors of COVID-19 severity: a systematic review and meta-analysis. F1000Res. 2020;9:1107.

22 Ramirez GA, Yacoub MR, Ripa M, et al. Eosinophils from physiology to disease: a comprehensive review. BioMed Res Int. 2018;2018, 9095275.

23 Daix T, Guerin E, Tavernier E, et al. Immature granulocytes: a risk factor of infection after cardiac surgery. Cytometry B Clin Cytom. 2018;94:887-894.

24 Lakota K, Zigon P, Mrak-Poljsak K, Rozman B, Shoenfeld Y, Sodin-Semrl S. Antibodies against acute phase proteins and their functions in the pathogenesis of disease: a collective profile of 25 different antibodies. Autoimmun Rev. 2011;10: 779-789. 